\title{
Variations
}

Variations

Revue internationale de théorie critique

15 | 2011

La haine

\section{Retour sur la notion d'expérience prolétarienne : Claude Lefort à Socialisme ou Barbarie}

Nicolas Poirier

\section{(2) OpenEdition}

Journals

Édition électronique

URL : http://journals.openedition.org/variations/105

DOI : 10.4000/variations. 105

ISSN : 1968-3960

Éditeur

Les amis de Variations

Édition imprimée

Date de publication : 1 mars 2011

\section{Référence électronique}

Nicolas Poirier, « Retour sur la notion d'expérience prolétarienne : Claude Lefort à Socialisme ou

Barbarie », Variations [En ligne], 15 | 2011, mis en ligne le 01 février 2012, consulté le 20 avril 2019

URL : http://journals.openedition.org/variations/105; DOI : 10.4000/variations.105

Les ami•e•s de Variations 


\section{Nicolas Poirier}

\section{Retour sur la notion d'expérience prolétarienne :}

\section{Claude Lefort à Socialisme ou Barbarie}

L'une des principales contributions de Claude Lefort au travail de réflexion critique développé par le groupe et la revue Socialisme ou Barbarie au cours des années 1950 a été de mettre en valeur l'idée d'expérience prolétarienne, qui est ce processus dynamique par lequel la classe ouvrière se constitue comme sujet historique, porteur d'un projet d'émancipation sociale dans le cadre d'une praxis conservant son autonomie par rapport à la théorie.

La notion d'expérience prolétarienne doit se comprendre dans le cadre de la critique développée par Lefort du marxisme sous sa forme la plus orthodoxe, et que celui-ci caractérise en tant que déterminisme objectiviste. Ainsi, à rebours du marxisme traditionnel, pour qui les luttes ouvrières ne constituent que le reflet d'un conflit objectif entre travail et Capital, conception impliquant de réduire l'action émancipatrice du prolétariat à un simple produit des lois économiques, Lefort entendait montrer que la classe ouvrière ne s'est pas bornée à réagir aux conditions qui lui avaient été faites, mais qu'elle s'est constituée comme sujet de son histoire au travers d'une expérience cumulative, et non en fonction seulement d'une situation objective'. C'est en fonction de cette créativité constitutive de l'expérience prolétarienne, qui reprend à son compte l'organisation de la production et se l'approprie par ses initiatives ${ }^{2}$, qu'on peut concevoir la possibilité d'un bouleversement révolutionnaire dont le prolétariat serait le sujet moteur.

\footnotetext{
1 Voir «L'expérience prolétarienne », Socialisme ou Barbarie, n¹1, 1952, repris dans Eléments d'une critique de la bureaucratie, Genève, Droz, 1971, rééd. « Tel-Gallimard », 1979, p. 73.

2 Voir ibid., p. 81.
} 
S’il est à ce titre juste de dire comme Marx et Engels que « l'histoire de toute société jusqu'à nos jours n'a été que l'histoire des luttes des classes ${ }^{1} »$, il est en même temps totalement erroné, d'après Lefort, de lire cette formule en des termes objectivistes, et de comprendre les rapports de classe comme de simples rapports de forces, du fait de certaines ambiguïtés présentes chez Marx lui-même. Contre cette conception déterministe des rapports de classe, Lefort cherche à montrer que le conflit de classes ne peut se réduire à la seule expression des rapports de force établis entre le Capital et le travail, et qui renverrait au conflit résultant de la contradiction entre l'essor des forces productives et l'état des rapports de production. Pas plus que la question de la politique n'est réductible à la question du pouvoir, encore moins à celle de la domination, le problème de la lutte des classes ne peut se concevoir sous l'angle des seuls rapports de force mettant aux prises des instances objectivement déterminées ${ }^{2}:$ l'analyse concrète des rapports sociaux ne peut avoir d'autre intérêt que de parvenir à saisir selon quelles modalités, des hommes placés dans les conditions objectives qui sont celles de la grande industrie, construisent une expérience commune par laquelle ils se constituent en sujet social doté d'une identité propre, et qui entretient avec sa propre histoire une réflexivité spécifique ${ }^{3}$. Bien entendu, fait remarquer Lefort, une analyse du mouvement ouvrier qui ne prendrait pas en compte l'existence d'une trame économique objective irréductible à l'intention des acteurs sociaux resterait partielle. Mais à l'inverse, une prise en compte de l'histoire de la classe ouvrière qui prétendrait réduire la situation de cette dernière au produit de déterminations objectives manquerait complètement son objet ${ }^{4}$.

Si Lefort critique donc avec virulence le marxisme orthodoxe, il n’évacue pas, loin de là, la pensée de Marx. Lefort articule en effet son propos en référence aux analyses développées par Marx sur la nature respective de la bourgeoisie et du prolétariat, qu'on ne saurait d'après lui placer dans

1 Manifeste communiste, trad. M. Rubel et L. Evrard, Euvres. Economie I, éd. M. Rubel, Paris, Gallimard, «Bibliothèque de la Pléiade », 1965, p. 161.

2 Voir «L'expérience prolétarienne », op. cit., p. 79.

3 Voir ibid., p. 79.

4 Voir ibid., p. 79-80. 
une situation symétrique. Il serait, de ce point de vue, fallacieux de réduire l'idée d'expérience prolétarienne au seul fait que les travailleurs auraient des intérêts communs contraires à ceux de la bourgeoisie. Certes, reconnaître, dans une perspective utilitariste, que les ouvriers, tout comme les bourgeois, ont des intérêts similaires peut sembler à première vue assez légitime : le fait d'avoir intérêt au plein emploi et à une hausse des salaires constitue un facteur déterminant dans la conscience de l'appartenance à une même classe sociale - mais, comme le fait remarquer Lefort, « ces intérêts sont, d'un certain point de vue, d'un autre ordre que [l'intérêt profond des ouvriers] qui est justement de ne pas être ouvriers ${ }^{1}$. » Le statut du prolétaire qui s'oppose au bourgeois est en effet totalement différent de celui du bourgeois qui cherche à augmenter son taux de profit ; possesseur d'une certaine marchandise, le capital, qu'il cherche à faire fructifier, le bourgeois se constitue immédiatement comme sujet de son action en tant que membre d'une classe sociale aux intérêts d'évidence similaires, alors que le prolétaire - en tant précisément qu'il a conscience de son exploitation - ne fait d'abord que réagir pour ainsi dire en tant qu'objet, en tout cas comme sujet aliéné qui ne se reconnaît pas dans le produit de son travail. Alors que le capitaliste est immédiatement face à lui-même comme un sujet qui se reconnaît dans son agir, le prolétaire se confronte à sa propre situation de classe en tant qu'il n'est rien positivement, et n'a d'abord pour projet rien d'autre que de nier la condition qui lui est faite en luttant contre l'exploitation. Ainsi, à en croire l'interprétation par Lefort du passage de L'Idéologie allemande où Marx et Engels cherchent à cerner les conditions de naissance de la bourgeoisie ${ }^{2}$, il est impossible de placer dans un rapport symétrique les situations respectives de la bourgeoisie et du prolétariat: la bourgeoisie est bourgeoisie dès qu'elle commence à étendre son mode de production dans la société féodale, son développement en tant que classe dominante ne faisant qu'actualiser des dispositions présentes dès l'origine; à l'inverse, le prolétaire ne devient pour ainsi dire prolétaire que lorsqu'il refuse la situation qui lui est faite et se met à lutter contre la classe bourgeoise, en visant non pas tant

Ibid., p. 77.

Voir K. Marx, Euvres III. Philosophie, éd. M. Rubel, Paris, Gallimard, «Bibliothèque de la Pléiade », 1982, p. 1106-1123. 
l'élimination de la bourgeoisie comme classe exploiteuse que l'abolition de toutes les classes ${ }^{1}$. Au fond, ce que cherche à faire ressortir Lefort à travers sa lecture de Marx, c'est que la bourgeoisie a besoin de reconnaitre la position du prolétaire en tant que prolétaire précisément, c'est-à-dire en tant que force de travail - c'est le fonctionnement normal du capitalisme -, là où le prolétaire ne devient sujet historique que par la négation, et de la classe exploiteuse qui lui extorque une plus-value, et de sa propre essence en tant que matière à exploitation ${ }^{2}$.

Contrairement donc à la bourgeoisie, dont le comportement et les aspirations découlent selon Marx de ses conditions normales d'existence, le prolétariat est soumis à la nécessité de lutter contre des conditions sociales qui tendent à le nier comme sujet, et c'est précisément dans cette lutte menée contre l'adversité, qu'elle prenne la forme de la nature hostile ou de rapports sociaux réifiés, que le prolétariat se constitue en tant que sujet social ; d'où, comme le fait remarquer Lefort, la nécessité de développer une approche spécifique du prolétariat, afin d'en cerner le développement à proprement parler subjectif ${ }^{3}$, et c'est précisément ce à quoi s'emploie Lefort en réfléchissant l'activité politique de la classe ouvrière à travers cette idée d'expérience prolétarienne. Toute classe sociale est certes dans l'obligation d'assimiler subjectivement les changements objectifs qui affectent sa place dans la structure sociale, mais c'est en un sens très particulier que la classe ouvrière se voit soumise à cette nécessité : se faire sujet historique et social dans le cadre d'une expérience commune, c'est pour la classe ouvrière naître en tant que telle ${ }^{4}$. La référence au jeune Marx est d'ailleurs mobilisée ici par Lefort contre le Marx du Capital, dont le défaut aurait consisté, d'après Lefort, à s'intéresser davantage aux formes objectives dans lesquelles se fixe l'action des

\footnotetext{
Voir « L'expérience prolétarienne », op. cit., p. 76-77.

2 Voir ibid., p. 77-78: «Le prolétariat ne s'affirme, en tant que classe autonome, en face de la classe bourgeoise, que lorsqu'il conteste son pouvoir, c'est-à-dire son mode de production, soit, concrètement, le fait même de l'exploitation; c'est donc son attitude révolutionnaire, qui constitue son attitude de classe. Ce n'est pas en étendant ses attributions économiques qu'il développe son sens de classe, mais en les niant radicalement pour instituer un nouvel ordre économique. »

3 Voir ibid., p. 78.

$4 \quad$ Voir C. Lefort, «Le prolétariat et sa direction », Socialisme ou Barbarie, n ${ }^{\circ}$, repris dans Eléments d'une critique de la bureaucratie, op. cit., p. 60-61.
} 
hommes, qu'à l'expérience humaine proprement dite à partir de laquelle peut se déployer l'ensemble de la structure matérielle et sociale ${ }^{1}$. Or s'il est clair que l'expérience des hommes est conditionnée par la réalité objective matérielle (sous forme notamment de techniques), en retour cette dernière résulte elle-même de l'expérience des hommes dont le travail transforme la nature et modifie la forme des rapports sociaux; ainsi que peut l'affirmer Lefort : "C'est en définitive l'analyse objective qui se subordonne à l'analyse concrète car ce ne sont pas les conditions mais les hommes qui sont révolutionnaires, et la question dernière est de savoir comment ils s'approprient et transforment leur situation ${ }^{2}$. " Il n'est en ce sens guère étonnant que Lefort ait pu concevoir, au cours de ces années, le projet d'une enquête auprès des ouvriers, qui leur donnerait la parole, afin de restituer « de l'intérieur » l'expérience concrète que ceux-ci peuvent faire de leur exploitation - l'étude du travail humain ne pouvant se réduire à l'évaluation objective des procédures techniques destinées à produire des marchandises, mais devant prendre en compte l'ensemble des rapports que l'ouvrier entretient à son travail, ainsi que les relations nouées aux autres dans le cadre d'une lutte plus générale contre l'exploitation ${ }^{3}$.

Sans doute doit-on lire dans les propos de Lefort une reprise de la formule de Marx «La classe ouvrière est révolutionnaire ou elle n'est rien ${ }^{4}$. » Par-là, Marx entendait en effet signifier que le prolétariat n'est pas une entité sociale que l'on peut définir par des caractéristiques sociologiques objectives, mais n'a d'existence que dans la seule mesure où celui-ci se constitue en tant que classe ouvrière, sujet porteur d'un projet d'émancipation qui nie ses conditions d'existence et se nie précisément comme prolétariat. La reconnaissance d'une asymétrie dans le conflit de classes n'est d'ailleurs pas un aspect secondaire de la pensée du «premier» Lefort, car c'est une idée qui sera encore très présente dans la suite de son travail, et occupera une place centrale dans le cadre de sa

\footnotetext{
Voir ibid., p. 80.

Ibid., p. 80.

Voir ibid., p. 86-97.

Voir la lettre de Marx à Schweitzer du 13 février 1865.
} 
réflexion sur la politique et sur le problème de la division du social : ainsi, dans son ouvrage sur Machiavel Le travail de l'ouvre Machiavel, Lefort cherchera à comprendre le sens du conflit entre les Grands et le peuple, non comme une simple lutte pour la domination, mais comme la confrontation de deux désirs irréductibles et placés dans une situation de totale asymétrie, le désir du peuple de ne pas être opprimé étant sans commune mesure avec le désir des Grands, puisque seul le premier ouvre vers un horizon politique qui rend légitime le désir de liberté et d'égalité1.

Cette d'idée d'expérience prolétarienne, par laquelle Lefort entend faire ressortir le processus de subjectivation propre à la classe ouvrière qui fait que celle-ci se constitue comme classe au sens politique, c'est-à-dire en tant que porteuse d'un projet révolutionnaire, anticipe sur les analyses d'Edward Palmer Thompson développées dans La formation de la classe ouvrière anglaise $^{2}$. Dans cet ouvrage novateur, à mi-chemin entre l'histoire politique et la sociologie critique, E. P. Thompson entendait apporter un éclairage nouveau sur la nature de la classe ouvrière, en tentant de frayer une voie entre l'approche positiviste issue des tendances empiristes de la sociologie traditionnelle, qui tend à nier l'idée même de classe sociale, et les conceptions du marxisme orthodoxe, incapables de saisir la formation de la classe ouvrière autrement que comme réaction mécanique à une situation d'exploitation ${ }^{3}$. A rebours donc de ces deux tendances réductrices, E. P. Thompson cherchait à mettre en valeur, non seulement la réalité de la classe ouvrière mais plus fondamentalement son caractère historique, permettant ainsi de faire ressortir la dynamique à partir de laquelle la classe se constitue comme un sujet politique à part entière qui, ainsi que l'affirme E. P. Thompson, se crée elle-même tout autant qu'on la crée ${ }^{4}$. Si Marx avait clairement montré qu'il n'y a pas en soi de classe ouvrière qui serait révolutionnaire, mais que sa constitution comme sujet politique est un processus qui s'inscrit dans une dynamique créatrice -

\footnotetext{
Voir Le travail de l'œuvre Machiavel, Paris, Gallimard, «Bibliothèque de philosophie », 1972, rééd. « Tel », 1986, p. 476-477.

2 Trad. G. Dauvé, M. Golaszewski et M.-N. Thibault, Paris, Gallimard/Le Seuil, « Hautes études », 1988.

Voir ibid., préface, p. 14-15.

$4 \quad$ Voir ibid., p. 174.
} 
qu'autrement dit le prolétariat n'est pas une réalité sociologique mais une instance politique ${ }^{1}-$, peut-être n'avait-il pas réussi à cerner avec assez de précision ce qui se joue fondamentalement dans le moment du passage de la «classe-en-soi» à la «classe-pour-soi $»^{2}$. Parler du passage à la « classe-pour-soi » comme d'un moment nécessaire n'implique d'ailleurs pas la reconnaissance de l'expérience prolétarienne comme telle, si ce passage doit se comprendre au sens où la conscience de classe est inculquée du dehors au prolétariat, réduit dans ces conditions au rang de « matériau humain brut, indifférencié et indéfinissable ${ }^{3}$.» Il faut donc prendre garde à ne pas tomber dans le piège qui aboutit à transformer la classe en chose, alors qu'elle est avant tout l'ensemble des rapports que celle-ci entretient à elle-même et à sa propre histoire ${ }^{4}$, et à substituer à l'expérience ouvrière par laquelle se forme le sujet politique « prolétariat», la théorie exacte fournie par le parti ${ }^{5}$. Une classe se définissant d'abord, comme le dit E. P. Thompson, par des hommes vivant leur propre histoire $^{6}$, il est impossible d'escamoter le rapport que la classe ouvrière entretient à son histoire, qu'elle fait autant qu'elle en est le produit, l'historicité formant la dimension constitutive de 1'expérience par laquelle le prolétariat prend conscience de lui-même en tant que classe ${ }^{7}$. Ce que manquent selon E. P. Thompson les approches sociologiques traditionnelles, qu'elles soient d'origine libérale ou marxiste, c'est précisément de pouvoir saisir le phénomène de l'historicité duquel les classes sociales sont inséparables : si l'on ne tient pas compte de l'expérience que font les hommes de leur rapport au travail et à la production, et qui est inscrite dans un temps relativement long, on ne peut plus appréhender la réalité d'une collectivité en train de se faire, mais on est conduit à ne voir de l'histoire qu'une suite de moments décousus où une multitude d'hommes vivent une multitude d'expériences sans lien véritable; comme peut l'écrire E. P. Thompson :

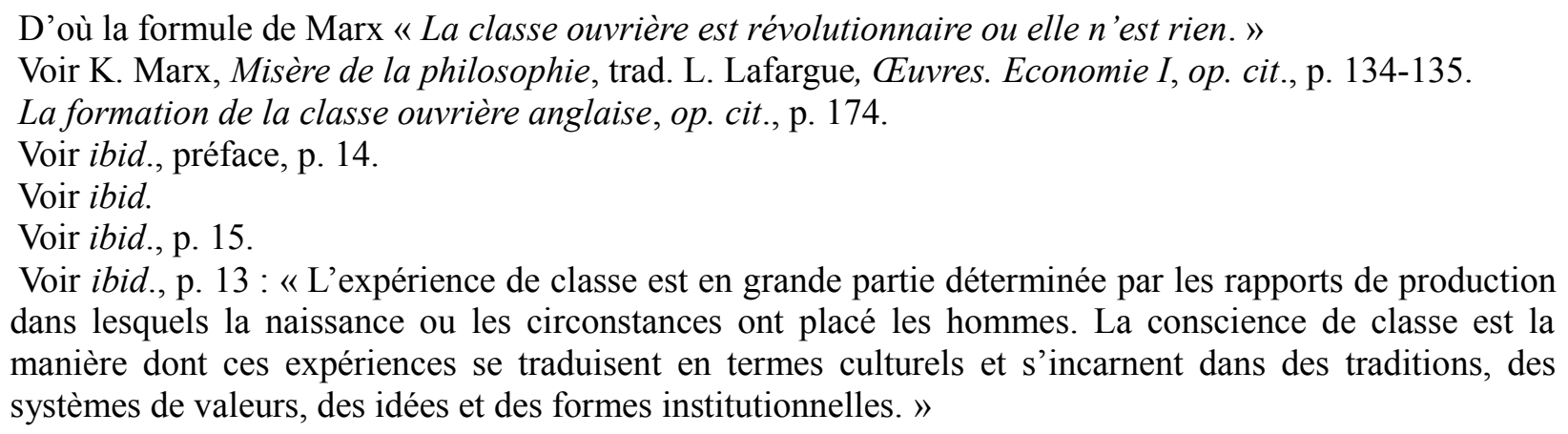
dans lesquels la naissance ou les circonstances ont placé les hommes. La conscience de classe est la manière dont ces expériences se traduisent en termes culturels et s'incarnent dans des traditions, des systèmes de valeurs, des idées et des formes institutionnelles. » 
«J'entends par classe un phénomène historique, unifiant des événements disparates et sans lien apparent, tant dans l'objectivité de l'expérience que dans la conscience. J'insiste sur le caractère historique du phénomène. Je ne conçois la classe ni comme une "structure" ni même comme une “catégorie”, mais comme quelque chose qui se passe en fait - et qui, on peut le montrer, s'est passé - dans les rapports humains ${ }^{1} . »$

Cette référence à la notion d'expérience prolétarienne permet de saisir le sens des nombreuses divergences entre Lefort et Castoriadis, divergences qui seront très vite apparues dans le cadre de leur collaboration à Socialisme ou Barbarie et qui renvoient, au-delà d'oppositions théoriques et philosophiques, à des problèmes d'ordre pratique, relatifs notamment à la question du parti, et plus généralement, des rapports entre la classe et l'organisation. Déjà réticent à l'idée d'une organisation qui aurait pour ambition de défendre des thèses programmatiques couvrant l'intégralité de la réalité sociale et politique, Lefort manifestera dans un autre texte de 1952, « Le prolétariat et sa direction $»^{2}$, son opposition aux conceptions de Castoriadis et de la majorité du groupe qui entendait se constituer en organisation politique.

Il s'agissait pour lui de remettre en cause l'idée même de parti, l'existence d'une direction révolutionnaire séparée constituant d'après la tendance minoritaire l'un des principaux facteurs de la « dégénérescence » bureaucratique. Les positions de Lefort sur ce point doivent se comprendre en rapport étroit avec sa conception de l'action ouvrière en tant qu'expérience politique par laquelle le prolétariat devient le sujet de son histoire, et on mesure bien l'importance de cette catégorie d'«expérience prolétarienne », puisqu'elle implique le fait de la créativité comme caractéristique intrinsèque du prolétariat, et ainsi, le refus d'une direction politique qui n'émanerait pas de ce processus d'auto-organisation. De ce point de vue, affirme Lefort, il est fallacieux de voir dans

\footnotetext{
I Ibid., p. 13. Castoriadis fait explicitement référence à E. P. Thompson dans « La question de l'histoire du mouvement ouvrier », 1973, L'expérience du mouvement ouvrier, 1, Paris, U.G.E., « 10-18 », 1974, p. 21 et p. 84-89.

2 Voir Eléments d'une critique de la bureaucratie, op. cit., p. 59-70.
} 
l'existence du parti un trait inhérent à l'expérience prolétarienne, et de considérer toute conception remettant en cause l'existence supposée nécessaire du parti comme une trahison des principes du marxisme ${ }^{1}$. La croyance en une telle nécessité résulte, d'après Lefort, d'une conception foncièrement abstraite de la politique révolutionnaire, incapable de prendre en compte l'effectivité de l'histoire en acte, et donc comme étrangère à l'expérience au travers de laquelle le prolétariat se constitue précisément en sujet révolutionnaire ${ }^{2}$.

Ce que veut signifier Lefort dans sa critique des positions défendues par la majorité du groupe Socialisme ou Barbarie concernant la nécessité d'une organisation partidaire, c'est que, conformément à la conception du jeune Marx, prolétariat et bourgeoisie n'ont pas un rapport similaire à la politique ; en effet, c'est précisément parce qu'elle est la classe détentrice du commandement de l'économie que la bourgeoisie peut s'aliéner dans une représentation politique au service de ses intérêts, la superstructure politique ne faisant dans son cas qu'exprimer la réalité profonde de l'infrastructure économique. Or ce qui vaut pour la bourgeoisie ne vaut d'aucune manière pour la classe ouvrière : celle-ci n'étant rien, ni sur le plan économique où elle est considérée comme une marchandise, ni sur le plan politique où elle est à l'origine privée de tout droit, elle n'a d'autre alternative que de se constituer comme sujet historique exprimant les intérêts de la société dans son ensemble ; n'ayant donc plus aucun intérêt partiel à faire valoir, le prolétariat n'a plus besoin d'en passer par l'intermédiaire d'une représentation politique, qui se révèle précisément à lui comme aliénation pure et simple ${ }^{3}$. Lefort reconnaît certes la nécessité stratégique d'une forme d'organisation politique, lorsque la conjoncture historique l'exige, c'est-à-dire juste avant la révolution, mais il serait selon lui impropre de caractériser comme une direction politique autonome ce regroupement spontané émanant de l'activité des organismes démocratiques, comme

Voir ibid., p. 62.

Voir ibid., p. 63.

Voir ibid., p. 66-67 : « ... Le prolétariat n'est rien d'objectif; il est une classe en qui l'économique et le politique n'ont plus de réalité séparée, qui ne se définit que comme expérience. C'est ce qui fait précisément son caractère révolutionnaire, mais ce qui indique son extrême vulnérabilité. C'est en tant que classe totale qu'il doit résoudre ses tâches historiques, et il ne peut remettre ses intérêts à une partie de lui détachée, car il n'a pas d'intérêts séparés de celui de la gestion de la société. » 
les Soviets - cette organisation n'ayant pour autre rôle que celui de faciliter la prise de pouvoir par le prolétariat, et devant donc logiquement s'abolir dans la perspective du gouvernement ouvrier ${ }^{1}$.

Lefort récuse au fond les termes du problème tel que le pose Castoriadis dans le sillage de Hegel $^{2}$ : poser selon lui la question de l'organisation à partir de la dialectique du particulier et de l'universel, c'est rester prisonnier d'une conception déterministe de la politique révolutionnaire, où sous prétexte de refuser les oppositions unilatérales et abstraites, on accorde finalement le primat à la catégorie de l'objet, pour dériver subrepticement vers une vision du parti comme seul détenteur légitime de la théorie politique juste. En témoigne le refus manifesté en permanence par Lefort de séparer la question politique à proprement parler de celle des luttes ouvrières elles-mêmes : dans un texte de $1958^{3}$, où une nouvelle fois celui-ci remet en question le rôle central de l'organisation, Lefort fait remarquer qu'il est abusif de réduire la question de l'unification au problème technique de la centralisation, en déduisant d'une telle nécessité l'existence du parti ${ }^{4}$. Que les travailleurs aient besoin de développer une perspective politique qui dépasse le cadre de leur seul lieu de travail est une chose, qu'on en tire la nécessité d'une organisation politique foncièrement autonome en est une autre : c'est d'après Lefort aux travailleurs eux-mêmes d'assurer la fonction de coordination des luttes dans le cadre d'un projet politique plus général, en assurant certes des relais en dehors du domaine de la production à proprement parler, mais en ne séparant jamais cette tâche de leurs activités politiques au sein de milieux professionnels qui ne pourront manquer de rester particuliers ${ }^{5}$. C'est en fait toujours en référence à l'autonomie de l'expérience prolétarienne qu'est réfutée la séparation de la classe et de l'organisation : ce n'est qu'à la condition d'assurer par lui-

1 Voir ibid., p. 68. L'exigence que marque dès ces années Lefort d'accorder sa primauté à l'agir effectif des hommes anticipe d'une certaine manière sur sa conception de la démocratie sauvage comme espace de luttes contre l'arbitraire de l'autorité étatique.

2 Voir C. Castoriadis, «Le parti révolutionnaire", Socialisme ou Barbarie, n², 1949, repris dans L'expérience du mouvement ouvrier, 1, op. cit., p. 121-143. Le texte de Castoriadis synthétise les positions de la majorité du groupe.

3 «Organisation et parti », Socialisme ou Barbarie, n²6, 1958, repris dans Eléments d'une critique de la bureaucratie, op. cit., p. 98-113.

4 Voir ibid., p. 110.

$5 \quad$ Voir ibid., p. 110. 
même les fonctions d'unification et d'universalisation inhérentes à l'action révolutionnaire que le prolétariat s'accomplit en tant que sujet politique ; puisque le prolétariat est privé dans les sociétés capitalistes d'institutions représentatives (ou en tout cas aliéné dans celles qu'il a pu créer), et que c'est un pur sujet qui ne dispose que de son expérience historique, il ne saurait exister de forme objective extérieure qui soit à la mesure de son être. En affirmant ainsi que la seule institution dont le prolétariat dispose, «c'est la révolution elle-même ${ }^{1}$, Lefort tend à réfuter par principe l'idée même de médiation, et donc celle de parti.

C'est au fond la problématique future du pouvoir et de son incarnation qui est en germe dans une telle conception : Lefort manifeste ici sa plus extrême méfiance envers les conceptions qui défendent l'existence du parti comme incarnation anticipée du pouvoir des travailleurs ${ }^{2}$. Sans doute Lefort voit-il dans cette tendance à l'incarnation la matrice du totalitarisme, et c'est aussi pour une part ce qui peut expliquer sa critique de l'idée révolutionnaire à partir des années 1960, et sa compréhension du phénomène démocratique comme renvoyant à une indétermination structurelle.

Il faut en tout cas saisir dans la divergence de vues entre Lefort et Castoriadis la racine de l'opposition qui se dessinera plus tard entre les deux penseurs et qui renvoie à deux conceptions bien différentes de la démocratie - l'objet de l'agir démocratique consistant davantage pour Lefort à contester le pouvoir, en revendiquant contre lui des droits, qu'à chercher à en démocratiser l'exercice effectif, comme le soutient Castoriadis ${ }^{3}$.

Nicolas Poirier

Ibid., p. 110.

2 Voir ibid., p. $110:$ «Que des minorités militantes fassent un travail révolutionnaire ne signifie nullement qu'un organisme puisse au sein de la société d'exploitation incarner en face du pouvoir bourgeois, sous une forme anticipée, grâce à la centralisation et à la rationalisation de ses activités, le pouvoir des travailleurs. » (Je souligne).

3 Voir sur ce point les remarques d'Antoine Chollet, « La question de la démocratie : Cornelius Castoriadis et Claude Lefort", dans Cornelius Castoriadis. Réinventer l'autonomie, collectif, dir. B. Bachofen, S. Elbaz, N. Poirier, Paris, éditions du Sandre, « Bibliothèque de philosophie contemporaine », 2008, p. 202. 